\title{
Optical Polarization Measurements of Pulsars
}

\author{
Stefan J. Wagner ${ }^{1}$ and W. Seifert, \\ Landessternwarte Heidelberg, Königstuhl, 69171 Heidelberg, Germany
}

\begin{abstract}
We investigate the polarization characteristics of optical emission from young pulsars. In a first attempt we search for polarization in images without time resolution in order to select suitable candidates for time-resolved studies. Polarization information constrains the nature of the faint optical emission of pulsars and will eventually allow to derive the topology of the field in those areas were optical radiation is emitted. We determine the polarization of the time-integrated optical emission of the SMC pulsar B0540-69 and the Vela pulsar B0833-45. We identify a new candidate optical counterpart for the young pulsar B1509-58 and find that the old candidate is unpolarized. The polarization of these three sources is rather high in view of the assumption that averaging over a putative swing of the polarization angle through 180 degrees will lead to depolarisation in the pulse-averaged signal.

We also detect high degrees of polarization in the synchrotron nebula surrounding the LMC pulsar B0540-69, indicating even further similarity to the Crab.
\end{abstract}

\section{Introduction}

At optical wavelengths pulsars are very faint but still significantly brighter than expected on the basis of extrapolations of the non-thermal radio emission. They are also much brighter than expected for the thermal emission from the cooling neutron star which is observed in the X-ray band. The radiation mechanisms responsible for the optical emission are not well understood. The low fluxes gave allowed detections of 8 pulsars with optical pulsation being detected in 4 of them. Spectra and even colours have been difficult to obtain and polarization studies have been even more difficult with $4 \mathrm{~m}$ class telescopes. It is important to distinguish between the various options proposed for optical emission and to constrain models of the radiation processes, and polarization characteristics are an important diagnostics. We started a programme to investigate polarization properties in the optical band with the VLT, and found polarized optical emission in three pulsars.

\footnotetext{
${ }^{1}$ Visiting Astronomer, ESO
} 


\section{Observation}

We observed several young pulsars with UT1 of the ESO-VLT, using the polarimetric mode of the FORS instrument. Ordinary and extraordinary beams are recorded simultaneously for several orientations of a beam-splitting Wollaston. Seeing conditions varied between 0.4 " and 1.0 " for the individual sources. The observations were carried out on April 13, 1999. During this first campaign all observations were carried out in a time-integrated mode. Four images with a different setting of a half-wave plate were obtained in each case. Total intensity maps are derived by resampling and shifting the eight images (two beams for four different angles) onto a common grid. In conditions of good seeing, this is equivalent to drizzling observations, improving the spatial sampling of the final maps. Stokes parameters are computed for each pixel from the ratios of the intensities in ordinary and extraordinary beam for the different settings of the half-wave plate. Further details on the observations and reduction procedures are discussed in forthcoming publications.

\section{PSR B 0540-693}

PSR B 0540-693 is a twin of the Crab Pulsar and Plerion in many ways. Unfortunately, its distance is 50 times larger. HST observations (Hill et al., 1997) isolated the pulsar at a slightly eccentric position within a diffuse remnant of 2 " diameter. This confirmed earlier identification based on phase resolved photometry that also resulted in the discovery of optical pulsation (Caraveo et al., 1992, Shearer et al., 1994).

\subsection{The synchrotron Nebula}

Figure 1 gives the total intensity map with polarization vectors superposed. The diffuse nebula is clearly polarized. This confirms single aperture polarimetry studies of pulsar+nebula by Chanan \& Helfand, 1990) and speculations (Chanan et al., 1984) that the nebula is due to synchrotron emission (with minor contributions of emission line filaments; such emission lines have been identified in spectroscopic analyzes). The maximum polarization $(20 \%)$ is at the rim of the diffuse nebulosity, possibly indicating the location of enhanced Doppler boosting. The orientation of the polarization vectors trace out a toroidal topology.

\subsection{The Pulsar}

Polarization from the pulsar itself was first explored by Middleditch et al., 1987 who determined upper limits in phase-resolved observations. Our images (figure 1) show a clear maximum of polarization at the location of the pulsar. Although one cannot rule out the possibility that this maximum is due to a knot of enhanced polarization within the synchrotron nebula, its precise spatial coincidence with the optical pulsar and the unresolved maximum in an image of polarized flux (different from the eccentric and spatially extended spot of maximum polarization at the rim of the synchrotron nebula) clearly suggests that the pulsar itself is polarized at a level of $5 \%$. This high degree of time-integrated polarization is a clear indication that the polarization angle does not exhibit a $180^{\circ}$ swing during the fairly symmetric optical pulse. 

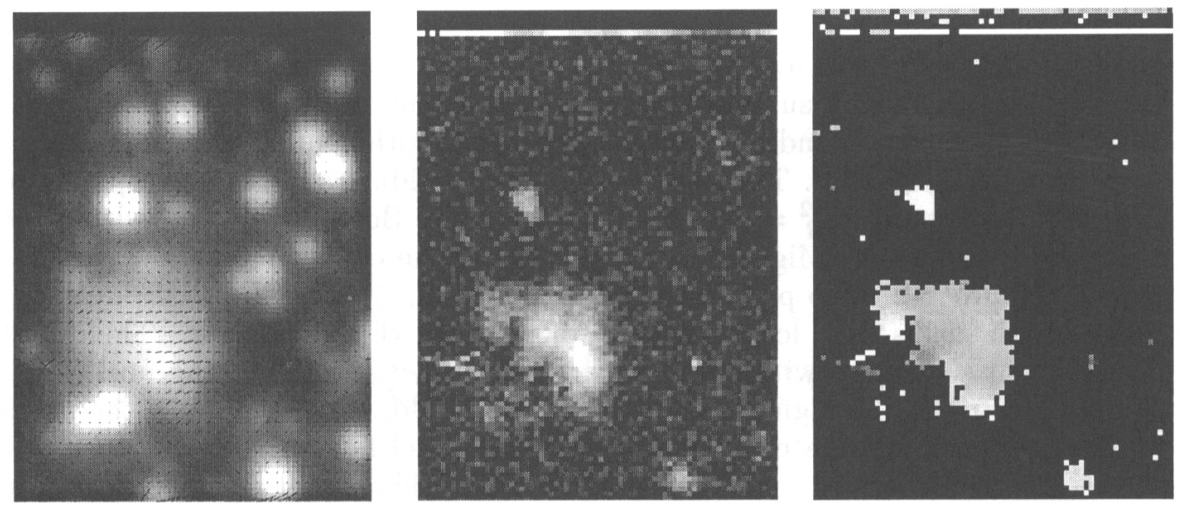

Figure 1. Polarization maps of the synchrotron nebula of PSR B 0540-693: Total flux with polarization vector plotted as overlay (left), polarized flux $(\mathrm{P} \times \mathrm{I}$, center $)$, note the foreground polarization being responsible for the signature towards the brightest foreground stars and the off-centered maximum. The right panel shows the polarization angle in a gray-scale representation.

\section{Vela (PSR B 0833-45)}

The optical counterpart of the Vela pulsar was identified by Lasker (1976). Timing observations by Wallace et al., 1977 and Manchester et al., 1980 derive an optical pulse profile clearly different from the radio pulse and an unpulsed contribution. A non-thermal origin of the optical emission was deduced by Nasuti et al., 1997, based on the rapid decrease of optical flux towards longer wavelengths but derive a softer slope in the R-I colour. The interstellar polarization is constant within the surroundings of the pulsar, and our images do not reveal extended polarized emission from an optical synchrotron-emitting plerion. The plerion, however, is larger than our field of view and difficult to detect in view of crowding of foreground stars and a comparatively strong polarization signal from the sky.

The total polarization of the time-integrated optical emission is $8.5 \pm 0.8 \%$, clearly strengthening the case for a non-thermal contribution to the optical emission. If the net polarization of the pulsed emission was zero, the unpulsed emission would be polarized at $17 \%(29 \%)$ for a pulse fraction of $0.5(0.7)$ at $660 \mathrm{~nm}$.

\section{PSR B 1509-58}

An optical candidate of this young pulsar was suggested by Caraveo et al., 1994 but was found to be several magnitudes brighter than expected. Searches for optical pulsations by Chakrabarty \& Kaspi, 1998 and Shearer et al., 1998 remained unsuccessful and suggested that the true optical counterpart of this pulsar was hidden among bright foreground stars in this crowded field. In our images the unblended stars on the total intensity map were found to have a seeing of 0.46 arcsec. Due to these good conditions it was possible to examine 
the candidate suggested by Caraveo et al. 1994 in detail. Subtraction of a pointspread function, which subtracts without significant residuals from a star 32" away from this pulsar candidate left two residuals, both being consistent in shape with an unblended PSF. The brightness of the residual sources corresponds to $\mathrm{m}_{R}^{\star 1}=25.7 \mathrm{mag}$ and $\mathrm{m}_{R}^{\star 2}=26.6 \mathrm{mag}$, respectively. Both objects fall within the error circle derived by Mignani et al., 1998, and the error circle of the brighter star 1 includes the radio position by Gaensler et al., 1999 .

Polarization peaks at a location $0.15 "$ offset from this candidate. This offset is consistent with zero within the uncertainties of determining the position of this source, given the high contrast (6 mag) and closeness $(0.65 ")$ of the much brighter original candidate. The integrated polarized flux corresponds to $10.4 \%$ of the total flux derived for the new candidate. This is still significant despite the systematics uncertainties involved in PSF subtraction. It is hence concluded, that this star is polarized, coincident in position with the radio pulsar, and hence a very likely optical counterpart of PSR 1509-58. Its total flux is well below the upper limits derived in timing studies by Mignani et al., 1998, Chakrabarty \& Kaspi, 1998, and Shearer et al., 1998. The high density of stars does not allow us to rule out the hypothesis that our new candidate is a distant unrelated star subject to strong interstellar polarization and ultimate confirmation requires timing analysis.

We do not detect optical signatures of an extended synchrotron nebulosity neither in total nor in polarized flux. In view of the very high surface density of stars, it is even more difficult to derive upper limits than in the case of the Vela pulsar.

Acknowledgments. SW is grateful to the conference organizers for a very interesting meeting. The authors thank Thomas Szeifert for valuable help in calibrating polarisation data.

\section{References}

Caraveo, P. et al., 1992 ApJ, 395, L103

Caraveo, P. et al., 1994 ApJ, 423, L125

Chanan, G.A. \& Helfand, D.J. 1990 ApJ, 352, 167

Chanan, G.A. et al., 1984 ApJ, 287, L23

Chakrabarty, D. \& Kaspi, V.M., 1998, ApJ498, L37

Gaenseler, B. et al., 1999, MNRAS, 305, 724

Hill, R.J. et al., 1997, ApJ486, L 99

Lasker, B. 1976, ApJ, 203, 193

Manchester, R.N. et al., 1980, MNRAS, 190,9P

Middleditch, J. et al., 1987, ApJ, 315, 142

Mignani, R.P. et al., 1998, Messenger, 94, 25

Nasuti, F.P. et al., 1997, A\&A, 323, 839

Shearer, A. et al., 1994, ApJ, 423, L51

Shearer, A. et al., 1998, A\&A, 333, L16

Wallace, P.T. et al., 1977, Nature, 266, 692 